\title{
Foreword:
}

\section{Special Feature for Detecting Afforestation, Reforestation, Deforestation using Remote Sensing and Geographical Information.}

\author{
Yoshio Awaya*
}

People have many opportunities to watch environmental TV programs and to read environmental articles in newspapers, and we become to be aware that human activities influence on the environment of the earth greatly. According to the third assessment report of the Intergovernmental Panel on Climate Changes (IPCC), global mean air temperature seems to have increased greatly since the 1910s along with the increasing greenhouse gases concentration. Because of this situation, plans for reducing greenhouse gas emissions were discussed at the Kyoto Conference organized by the parties to the United Nations Framework Convention on Climatic Change (UNFCCC) in 1997. The agreements were documented as the Kyoto Protocol to UNFCCC. Reducing emissions of six greenhouse gases including atmospheric carbon dioxide has been obligated to the parties included in Annex I. Japan has been obligated a six percent reduction from the base line emission in 1990 during 2008 and 2012 . The Kyoto Protocol defines that "The net changes in greenhouse gas emissions by sources and removals by sinks resulting from direct human-induced land-use change and forestry activities, limited to afforestation, reforestation and deforestation (ARD) since 1990, measured as verifiable changes in carbon stocks in each commitment period, shall be used to meet the commitments." The Forestry Agency has sights on achieving 3.9\% of sinks to the base line emission by results from forestry activities in Japan. However, the removals of greenhouse gas emissions shall be reported in a transparent and verifiable manner.

Area of ARD is mandatory and basic information in the national report of greenhouse gas emissions. Due to lack of political information at the time of 1990, remotely sensed images are the most useful and reliable information to know land-covers at that time. Therefore, change detection using images is a practical method to identify ARD. However, there are some problems to be solved to use remotely sensed images in ARD detection. Although ARD is defined by land-use change, images provide land-cover information. Seasonal and successional vegetation changes may disturb in identifying ARD by image interpretation. The Forestry Agency has planned to detect ARDs by image interpretation and had started "the Forest Carbon Account and Utilization System Buildup Project" to develop methods for reports corresponding to the Kyoto Protocol Article 3.3 and 3.4. ARD detection and forest area mapping methods were tested using remotely sensed images and geographical information, and a visual interpretation method was proposed for ARD detection in the project.

This special feature introduces developed methods and critical problems to be overcome in ARD detection and forest area mapping. The articles are results of "the Forest Carbon Account and Utilization System Buildup Project". I greatly appreciate for the efforts of authors, administrative support by the staff of Forestry Agency and financial support by the Forestry Agency.

* Forestry and Forest Products Research Institute 